\title{
Online Supplement for Calendar-based Age Replacement Policy with Dependent Renewal Cycles
}

Maliheh Aramon Bajestani and Dragan Banjevic

Department of Mechanical \& Industrial Engineering, University of Toronto, Toronto, Ontario, Canada $\{$ maramon,banjev\}@mie.utoronto.ca

\section{A Proofs}

Lemma 1 If $0<\lambda \leq R\left(t_{p}+2 \Delta\right)$, then our chain $\left\{X_{n}\right\}$ is $(0, \lambda, \varphi, 1)$ recurrent.

Proof. Defining $p_{n}(x)=\mathbb{P}\left\{X_{i} \neq 0,1 \leq i \leq n \mid X_{0}=x\right\}$, condition (i), $\mathbb{P}\left\{X_{n} \in A\right.$ for some $n \geq 1 \mid X_{0}=$ $x\}=1, \forall x \in S$, is equivalent to $\lim _{n \rightarrow \infty} p_{n}(x)=0, \forall x \in S$. Let $p_{n}^{+}=\sup _{0 \leq x<\Delta} p_{n}(x)$; using the Markovian property, we have $p_{n}(x)=\int_{0}^{\Delta} p(x, y) p_{n-1}(y) d y \leq p_{n-1}^{+} \int_{0}^{\Delta} p(x, y) d y=p_{n-1}^{+}(1-p(x, 0))$ or $p_{n}^{+} \leq p_{n-1}^{+}(1-$ $p(x, 0)) \leq p_{1}^{+}(1-p(x, 0))^{n-1} \leq(1-p(x, 0))^{n-1}$. Further, As mentioned in Section $2, p(x, 0)=R(n(x) \Delta-$ $x)>R(n(\Delta) \Delta)>R\left(t_{p}+2 \Delta\right), \forall x$. Therefore, $p_{n}^{+} \leq(1-p(x, 0))^{n-1} \leq\left(1-R\left(t_{p}+2 \Delta\right)\right)^{n-1}$. Based on our assumption, since $R\left(t_{p}+2 \Delta\right)>0$, we will have $\lim _{n \rightarrow \infty} p_{n}(x)=0, \forall x \in S$.

Condition (ii), $\mathbb{P}\left\{X_{n_{0}} \in B \mid X_{0}=x\right\} \geq \lambda \varphi(B), \forall x \in A$ and $B \subset A$, is equivalent to $\mathbb{P}\left\{X_{1}=0 \mid X_{0}=0\right\} \geq$ $\lambda$ in our problem. We have $\mathbb{P}\left\{X_{1}=0 \mid X_{0}=0\right\}=p(0,0)$ where $p(0,0)=R(n(0) \Delta)>R\left(t_{p}+2 \Delta\right) \geq \lambda>0$.

Since both conditions (i) and (ii) are satisfied, the Markov chain $\left\{X_{n}\right\}$ is recurrent.

Lemma 2 In our recurrent Markov chain $\left\{X_{n}\right\}, \mathbb{E}\left[N_{1} \mid X_{0}=0\right]<\infty$.

Proof. We have:

$$
\begin{aligned}
\mathbb{E}\left[N_{1} \mid X_{0}=0\right] & =\mathbb{E}\left[\sum_{i=0}^{\infty} \mathbb{1}\left\{N_{1}>i\right\} \mid X_{0}=0\right]=1+\sum_{i=1}^{\infty} \mathbb{E}\left[\mathbb{1}\left\{N_{1}>i\right\} \mid X_{0}=0\right] \\
& =1+\sum_{i=1}^{\infty} \mathbb{P}\left\{X_{1} \neq 0, X_{2} \neq 0, \ldots, X_{i} \neq 0 \mid X_{0}=0\right\} .
\end{aligned}
$$

As mentioned in the proof of Lemma $1, p_{n}(0)=\mathbb{P}\left\{X_{i} \neq 0,1 \leq i \leq n \mid X_{0}=0\right\} \leq(1-p(0,0))^{n-1}$; therefore, we have

$$
\mathbb{E}\left[N_{1} \mid X_{0}=0\right]=1+\sum_{i=1}^{\infty} p_{i}(0) \leq \sum_{i=1}^{\infty}(1-p(0,0))^{i-1}=\frac{1}{p(0,0)} .
$$

As already shown in the proof of Lemma $1, p(0,0)>0$. Therefore, $\mathbb{E}\left[N_{1} \mid X_{0}=0\right]<\infty$, which completes the proof. 
Theorem 1 The expected total cost per time unit for an infinite time span equals:

$$
\lim _{t \rightarrow \infty} \frac{\mathbb{E}[C(t)]}{t}=\frac{\mathbb{E}\left[C_{0} \mid X_{0}=0\right] p(0)+\int_{S \backslash\{0\}} \mathbb{E}\left[C_{0} \mid X_{0}=y\right] p(y) d y}{\mathbb{E}\left[L_{0} \mid X_{0}=0\right] p(0)+\int_{S \backslash\{0\}} \mathbb{E}\left[L_{0} \mid X_{0}=y\right] p(y) d y} .
$$

Proof. To prove this theorem, we use similar ideas as used by Athreya et al. (1978) and Athreya and Ney (1978).

As mentioned in Section 2, we have:

$$
\lim _{t \rightarrow \infty} \frac{\mathbb{E}[C(t)]}{t}=\frac{\mathbb{E}\left[\sum_{n=0}^{N_{1}-1} C_{n} \mid X_{0}=0\right]}{\mathbb{E}\left[\sum_{n=0}^{N_{1}-1} L_{n} \mid X_{0}=0\right]}
$$

We then have:

$$
\mathbb{E}\left[\sum_{n=0}^{N_{1}-1} C_{n} \mid X_{0}=0\right]=\mathbb{E}\left[\sum_{n=0}^{\infty} C_{n} \mathbb{1}\left\{N_{1}>n\right\} \mid X_{0}=0\right]=\mathbb{E}\left[C_{0} \mid X_{0}=0\right]+\mathbb{E}\left[\sum_{n=1}^{\infty} C_{n} \mathbb{1}\left\{N_{1}>n\right\} \mid X_{0}=0\right] .
$$

Since $\left\{N_{1}>n\right\}=\left\{X_{1} \neq 0, \ldots, X_{n-1} \neq 0, X_{n} \neq 0\right\}$,

$\mathbb{E}\left[\sum_{n=1}^{\infty} C_{n} \mathbb{1}\left\{N_{1}>n\right\} \mid X_{0}=0\right]=\sum_{n=1}^{\infty} \mathbb{E}\left[C_{n} \mathbb{1}\left\{X_{1} \neq 0, \ldots, X_{n-1} \neq 0, X_{n} \neq 0\right\} \mid X_{0}=0\right]$.

Conditioning on $\left(X_{1}, X_{2}, \ldots, X_{n-1}, X_{n}\right)$, we have

$$
\begin{aligned}
\mathbb{E}\left[\sum_{n=1}^{\infty} C_{n} \mathbb{1}\left\{N_{1}>n\right\} \mid X_{0}=0\right] & =\sum_{n=1}^{\infty} \mathbb{E}\left[\mathbb{E}\left[C_{n} \mathbb{1}\left\{X_{1} \neq 0, \ldots, X_{n-1} \neq 0, X_{n} \neq 0\right\} \mid X_{0}=0, X_{1}, \ldots, X_{n-1}, X_{n}\right]\right] \\
& =\sum_{n=1}^{\infty} \mathbb{E}\left[\mathbb{1}\left\{X_{1} \neq 0, \ldots, X_{n-1} \neq 0, X_{n} \neq 0\right\} \mathbb{E}\left[C_{n} \mid X_{n}\right] \mid X_{0}=0\right] \\
& =\sum_{n=1}^{\infty} \int_{S \backslash\{0\}} \int_{S \backslash\{0\}} \mathbb{E}\left[C_{n} \mid X_{n}=y\right] \mathbb{E}\left[\mathbb{1}\left\{X_{1} \neq 0, \ldots, X_{n-1}=x\right\} \mid X_{0}=0\right] p(x, y) d x d y .
\end{aligned}
$$

Since the distribution of $C_{n}$ only depends on $X_{n}$, we have

$$
=\int_{S \backslash\{0\}} \int_{S \backslash\{0\}} \mathbb{E}\left[C_{0} \mid X_{0}=y\right] \sum_{n=1}^{\infty} \mathbb{P}\left\{X_{1} \neq 0, \ldots, X_{n-1}=x \mid X_{0}=0\right\} p(x, y) d x d y
$$

Changing $n-1$ to $n$, we have

$$
=\int_{S \backslash\{0\}} \int_{S \backslash\{0\}} \mathbb{E}\left[C_{0} \mid X_{0}=y\right] \sum_{n=0}^{\infty} \mathbb{P}\left\{X_{1} \neq 0, \ldots, X_{n}=x \mid X_{0}=0\right\} p(x, y) d x d y .
$$


Using the definition of $v($.$) , we have$

$$
\begin{aligned}
& =\int_{S \backslash\{0\}} \mathbb{E}\left[C_{0} \mid X_{0}=y\right] \int_{S \backslash\{0\}} \sum_{n=0}^{N_{1}-1} \mathbb{E}\left[\mathbb{1}\left\{X_{n} \in(x, x+d x)\right\} \mid X_{0}=0\right] d x p(x, y) d y \\
& =\int_{S \backslash\{0\}} \mathbb{E}\left[C_{0} \mid X_{0}=y\right] \int_{S \backslash\{0\}} d \nu(x) p(x, y) d y .
\end{aligned}
$$

Since $v($.$) is an invariant measure, then$

$$
=\int_{S \backslash\{0\}} \mathbb{E}\left[C_{0} \mid X_{0}=y\right] d v(y) .
$$

As $d v(y)=g(y) d y$ (Wang and Banjevic, 2012), we finally have

$$
\mathbb{E}\left[\sum_{n=0}^{N_{1}-1} C_{n} \mid X_{0}=0\right]=\mathbb{E}\left[C_{0} \mid X_{0}=0\right]+\int_{S \backslash\{0\}} \mathbb{E}\left[C_{0} \mid X_{0}=y\right] g(y) d y .
$$

Using the same reasoning as above, we have $\mathbb{E}\left[\sum_{n=0}^{N_{1}-1} L_{n} \mid X_{0}=0\right]=\mathbb{E}\left[L_{0} \mid X_{0}=0\right]+\int_{S \backslash\{0\}} \mathbb{E}\left[L_{0} \mid X_{0}=\right.$ $y] g(y) d y$; then,

$\lim _{t \rightarrow \infty} \frac{\mathbb{E}[C(t)]}{t}=\frac{\mathbb{E}\left[C_{0} \mid X_{0}=0\right]+\int_{S \backslash\{0\}} \mathbb{E}\left[C_{0} \mid X_{0}=y\right] g(y) d y}{\mathbb{E}\left[L_{0} \mid X_{0}=0\right]+\int_{S \backslash\{0\}} \mathbb{E}\left[L_{0} \mid X_{0}=y\right] g(y) d y}=\frac{\mathbb{E}\left[C_{0} \mid X_{0}=0\right] p(0)+\int_{S \backslash\{0\}} \mathbb{E}\left[C_{0} \mid X_{0}=y\right] p(y) d y}{\mathbb{E}\left[L_{0} \mid X_{0}=0\right] p(0)+\int_{S \backslash\{0\}} \mathbb{E}\left[L_{0} \mid X_{0}=y\right] p(y) d y}$.

Proposition 1 Letting $\mu=\int_{0}^{\infty} R(t) d t$, for $\Delta>0, \varlimsup_{t_{p} \rightarrow \infty} G\left(t_{p}\right) / H\left(t_{p}\right) \leq\left(c_{f}+c_{s}\right) / \mu+2 c_{s} / \Delta$.

Proof. For $(k-1) \Delta<t_{p} \leq k \Delta, k \geq 1$, we have:

$$
\begin{aligned}
G\left(t_{p}\right)=c_{f} & +c_{s} \sum_{n=1}^{k}\left[R(n \Delta) p(0)+\int_{0}^{\Delta} R(n \Delta-x) p(x) d x\right]+\left(c_{p}-c_{f}\right)\left[R(k \Delta) p(0)+\int_{0}^{k \Delta-t_{p}} R(k \Delta-x) p(x) d x\right] \\
& +\left(c_{s}+c_{p}-c_{f}\right) \int_{k \Delta-t_{p}}^{\Delta} R((k+1) \Delta-x) p(x) d x
\end{aligned}
$$

Since $\sum_{n=1}^{k}\left[R(n \Delta) p(0)+\int_{0}^{\Delta} R(n \Delta-x) p(x) d x\right] \leq \sum_{n=1}^{k}[R(n \Delta)+R((n-1) \Delta)], \int_{0}^{k \Delta-t_{p}} R(k \Delta-x) p(x) d x \leq$ $\int_{0}^{\Delta} p(x) d x R\left(t_{p}\right) \leq R\left(t_{p}\right)$, and $\int_{k \Delta-t_{p}}^{\Delta} R((k+1) \Delta-x) p(x) d x \leq \int_{0}^{\Delta} p(x) d x R\left(t_{p}\right) \leq R\left(t_{p}\right)$, we have:

$$
\varlimsup_{t_{p} \rightarrow \infty} G\left(t_{p}\right)=c_{f}+c_{s} \sum_{n=1}^{\infty}[R(n \Delta)+R((n-1) \Delta)]+0+0 \leq c_{f}+c_{s}\left(1+2 \frac{\mu}{\Delta}\right)
$$


Furthermore, for $(k-1) \Delta<t_{p} \leq k \Delta, k \geq 1$, we have:

$$
\begin{aligned}
H\left(t_{p}\right)= & \int_{0}^{k \Delta} R(t) d t p(0)+\int_{0}^{\Delta}\left[\int_{0}^{k \Delta-x} t f(t) d t\right] p(x) d x+\int_{0}^{k \Delta-t_{p}} k \Delta R(k \Delta-x) p(x) d x \\
& +\int_{k \Delta-t_{p}}^{\Delta}\left[\int_{k \Delta-x}^{(k+1) \Delta-x} t f(t) d t+(k+1) \Delta R((k+1) \Delta-x)\right] p(x) d x . \\
= & \int_{0}^{k \Delta} R(t) d t-\int_{0}^{\Delta}\left[k \Delta R(k \Delta)+\int_{k \Delta-x}^{k \Delta} t f(t) d t\right] p(x) d x+\int_{0}^{k \Delta-t_{p}} k \Delta R(k \Delta-x) p(x) d x \\
& +\int_{k \Delta-t_{p}}^{\Delta}\left[\int_{k \Delta-x}^{(k+1) \Delta-x} t f(t) d t+(k+1) \Delta R((k+1) \Delta-x)\right] p(x) d x .
\end{aligned}
$$

Since $\int_{0}^{\Delta}\left[k \Delta R(k \Delta)+\int_{k \Delta-x}^{k \Delta} t f(t) d t\right] p(x) d x \leq\left[\left(t_{p}+\Delta\right) R\left(t_{p}\right)+\int_{t_{p}-\Delta}^{t_{p}+\Delta} t f(t) d t\right], \int_{0}^{k \Delta-t_{p}} k \Delta R(k \Delta-x) p(x) d x \leq$ $\left(t_{p}+\Delta\right) R\left(t_{p}\right)$, and $\int_{k \Delta-t_{p}}^{\Delta}\left[\int_{k \Delta-x}^{(k+1) \Delta-x} t f(t) d t+(k+1) \Delta R((k+1) \Delta-x)\right] p(x) d x \leq\left[\int_{t_{p}-\Delta}^{t_{p}+\Delta} t f(t) d t+\left(t_{p}+2 \Delta\right) R\left(t_{p}\right)\right]$, we have $\varlimsup_{t_{p} \rightarrow \infty} \int_{0}^{\Delta}\left[k \Delta R(k \Delta)+\int_{k \Delta-x}^{k \Delta} t f(t) d t\right] p(x) d x=0, \varlimsup_{t_{p} \rightarrow \infty} \int_{0}^{k \Delta-t_{p}} k \Delta R(k \Delta-x) p(x) d x=0$, and $\varlimsup_{t_{p} \rightarrow \infty} \int_{k \Delta-t_{p}}^{\Delta}\left[\int_{k \Delta-x}^{(k+1) \Delta-x} t f(t) d t+(k+1) \Delta R((k+1) \Delta-x)\right] p(x) d x=0$. As all three expressions are positive, their lower limits also equal 0 . Therefore, their limits equal 0 and we have:

$$
\lim _{t_{p} \rightarrow \infty} H\left(t_{p}\right)=\int_{0}^{\infty} R(t) d t-0+0+0=\mu .
$$

Finally, $\varlimsup_{t_{p} \rightarrow \infty} G\left(t_{p}\right) / H\left(t_{p}\right) \leq\left(c_{f}+c_{s}\right) / \mu+2 c_{s} / \Delta$.

Proposition 2 If $t_{p}<\Delta$ (modified block replacement policy), the expected cost per time unit (Equation 5) reduces to

$$
\frac{c_{p}+c_{f} \int_{0}^{\Delta} g(y) d y+c_{s} \int_{\Delta-t_{p}}^{\Delta} R(\Delta-y) g(y) d y}{t\left[1+\int_{\Delta-t_{p}}^{\Delta} R(\Delta-y) g(y) d y\right]}
$$

Proof. If $t_{p}<\Delta$, the one-step transition kernel of the Markov Chain can be written as below:

$$
p(x, 0)=\left\{\begin{array}{ll}
R(\Delta-x) & , x \leq \Delta-t_{p} \\
R(2 \Delta-x) & , x>\Delta-t_{p}
\end{array},\right.
$$

and for $y>0$ : 


$$
p(x, y)=\left\{\begin{array}{ll}
0 & , y<x \leq \Delta-t_{p} \\
f(y-x) & , x \leq \min \left(y, \Delta-t_{p}\right) \\
f(\Delta-x+y) & , x>\max \left(y, \Delta-t_{p}\right) \\
f(y-x)+f(\Delta-x+y) & , \Delta-t_{p}<x \leq y
\end{array} .\right.
$$

The integral equation of the $g()$ measure (Equation 4) can be then rewritten as follows:

$$
g(y)=f(y)+\int_{0}^{y} f(y-u) g(u) d u+\int_{\Delta-t_{p}}^{\Delta} f(\Delta-u+y) g(u) d u .
$$

Let us introduce

$$
\begin{aligned}
\mathcal{H}(z) & =R(z)+\int_{0}^{z} R(z-u) g(u) d u+\int_{\Delta-t_{p}}^{\Delta} R(\Delta-u+z) g(u) d u \text {. Then, } \\
\frac{\partial \mathcal{H}(z)}{\partial z} & =-\left[f(z)+\int_{0}^{z} f(z-u) g(u) d u-g(z)+\int_{\Delta-t_{p}}^{\Delta} R(\Delta-u+z) g(u) d u\right]=-[g(z)-g(z)]=0 .
\end{aligned}
$$

Therefore, $\mathcal{H}(z)=\mathcal{H}(0)=1+\int_{\Delta-t_{p}}^{\Delta} R(\Delta-u) g(u) d u$. Let us further introduce $\mathcal{G}(z)=\int_{0}^{z} R(u) d u$ and $\mathcal{J}(z)=\mathcal{G}(z)+\int_{0}^{z} \mathcal{G}(z-u) g(u) d u+\int_{\Delta-t_{p}}^{\Delta} \mathcal{G}(\Delta-u+z) g(u) d u$. We then have

$$
\frac{\partial \mathcal{J}(z)}{\partial z}=R(z)+\int_{0}^{z} R(z-u) g(u) d u+\int_{\Delta-t_{p}}^{\Delta} R(\Delta-u+z) g(u) d u=\mathcal{H}(z)=1+\int_{\Delta-t_{p}}^{\Delta} R(\Delta-u) g(u) d u .
$$

Therefore,

$$
\mathcal{J}(z)=\left[1+\int_{\Delta-t_{p}}^{\Delta} R(\Delta-u) g(u) d u\right] z+\int_{\Delta-t_{p}}^{\Delta} \mathcal{G}(\Delta-u) g(u) d u
$$

Using the one-step transition kernels, the expected cost per time unit ratio (Equation 5) equals $\left[c_{f} \mathcal{A}+c_{p} \mathcal{B}+c_{s}\left(\mathcal{B}+\int_{\Delta-t_{p}}^{\Delta} R(\Delta-y) g(y) d y\right)\right] / C$, where

$$
\begin{aligned}
\mathcal{A} & =(1-R(\Delta))+\int_{0}^{\Delta-t_{p}}(1-R(\Delta-u)) g(u) d u+\int_{\Delta-t_{p}}^{\Delta}(1-R(2 \Delta-u)) g(u) d u, \\
\mathcal{B} & =R(\Delta)+\int_{0}^{\Delta-t_{p}} R(\Delta-u) g(u) d u+\int_{\Delta-t_{p}}^{\Delta} R(2 \Delta-u) g(u) d u, \\
C & =\int_{0}^{\Delta} R(z) d z+\int_{0}^{\Delta-t_{p}} \int_{0}^{\Delta-u} R(z) d z g(u) d u+\int_{\Delta-t_{p}}^{\Delta} \int_{0}^{2 \Delta-u} R(z) d z g(u) d u .
\end{aligned}
$$


Using the functions defined above, it is easy to see

$$
\begin{aligned}
& \mathcal{A}=1+\int_{0}^{t} g(u) d u-\left[\mathcal{H}(t)-\int_{\Delta-t_{p}}^{\Delta} R(\Delta-u) g(u) d u\right]=\int_{0}^{t} g(u) d u, \\
& \mathcal{B}=\mathcal{H}(t)-\int_{\Delta-t_{p}}^{\Delta} R(\Delta-u) g(u) d u=1, \\
& C=\mathcal{J}(t)-\int_{\Delta-t_{p}}^{\Delta} \mathcal{G}(\Delta-u) g(u) d u=\left[1+\int_{\Delta-t_{p}}^{\Delta} R(\Delta-u) g(u) d u\right] t .
\end{aligned}
$$

Finally, the expected cost per time unit is as the given equation in the proposition.

\section{B The Ergodic Measure for the Exponential and the Erlang Time to Failure Distributions}

If $t_{p}<\Delta$, the ergodic measure $p()$ can be derived analytically for the Exponential and Erlang time to failure distributions. As mentioned in the proof of Proposition 2, for $t_{p}<\Delta$, we have $g(y)=f(y)+\int_{0}^{y} f(y-u) g(u) d u+\int_{\Delta-t_{p}}^{\Delta} f(\Delta-u+y) g(u) d u$.

Exponential Distribution Let us assume that $f(y)=\lambda e^{-\lambda y}$. The above equation reduces to $g(y)=$ $\lambda e^{-\lambda y}+\int_{0}^{y} \lambda e^{-\lambda(y-u)} g(u) d u+\int_{\Delta-t_{p}}^{\Delta} \lambda e^{-\lambda(\Delta-u+y)} g(u) d u$. Multiplying this by $e^{\lambda y}$ and defining $\tilde{g}(y)=e^{\lambda y} g(y)$, we have:

$$
\begin{aligned}
\tilde{g}(y) & =\lambda+\lambda \int_{0}^{y} \tilde{g}(u) d u+\lambda e^{-\lambda \Delta} \int_{\Delta-t_{p}}^{\Delta} \tilde{g}(u) d u, \\
\frac{\partial \tilde{g}(y)}{\partial y} & =\lambda \tilde{g}(y) \Rightarrow \tilde{g}(y)=\tilde{g}(0) e^{\lambda y} .
\end{aligned}
$$

Therefore, $g(y)=\tilde{g}(0)$. Finding $\tilde{g}(0)$ from $\tilde{g}(0)=\lambda+\lambda e^{-\lambda \Delta} \int_{\Delta-t_{p}}^{\Delta} \tilde{g}(u) d u$, we have:

$$
g(y)=\lambda e^{\lambda t_{p}}, \quad p(0)=\frac{1}{1+\lambda \Delta e^{\lambda t_{p}}}, \quad p(y)=\frac{\lambda e^{\lambda t_{p}}}{1+\lambda \Delta e^{\lambda t_{p}}}, 0<y<\Delta
$$

Erlang Distribution For the Erlang distribution where $f(y)=\lambda^{k} y^{k-1} e^{-\lambda y} /(k-1)$ !, it is easy to see that $\partial^{k} \tilde{g}(y) / \partial y^{k}=\lambda^{k} \tilde{g}(y)$. This is a $k$-th order linear differential equation whose general solution can be found by solving the characteristic equation $r^{k}-\lambda^{k}=0$ (Boyce and DiPrima, 2012). For example, if $\lambda=1$ and $k=2$, it can be shown that $p(0)=1 /\left[1+c_{1} \Delta+\frac{c_{2}}{2}\left(1-e^{-2 \Delta}\right)\right]$ and $p(y)=$ $\left[c_{1}+c_{2} e^{-2 y}\right] /\left[1+c_{1} \Delta+\frac{c_{2}}{2}\left(1-e^{-2 \Delta}\right)\right]$, where $c_{1}$ and $c_{2}$ can be found using $\tilde{g}^{\prime}(y)=c_{1} e^{y}-c_{2} e^{-y}$ and $1=R(\Delta)+\int_{0}^{\Delta-t_{p}} R(\Delta-u) g(u) d u+\int_{\Delta-t_{p}}^{\Delta} R(2 \Delta-u) g(u) d u$. 


\section{Analyzing the Calendar-based Age Replacement Policy using Markov Chain of Ages}

In Section 2, we defined $X_{n}$ to be the $n$-th forward time. Let us now define a new Markov chain where $X_{n}$ represents the age of the component at fixed times $\Delta^{+},(2 \Delta)^{+},(3 \Delta)^{+}, \ldots$ where $0 \leq X_{n} \leq$ $t_{p}$. Whenever $X_{n}=0$, a preventive renewal has occurred at checking points and the Markov chain therefore restarts itself. In this definition, $\left\{X_{n}=0\right\}$ is a candidate for the regeneration set $A$. Following the same analysis as presented in Sections 3 and 4 , it is easy to see that Theorem 1 can be written as $\lim _{t \rightarrow \infty} \mathbb{E}[C(t)] / t=\left[\mathbb{E}\left[C_{0} \mid X_{0}=0\right] p(0)+\int_{0}^{t_{p}} \mathbb{E}\left[C_{0} \mid X_{0}=y\right] p(y) d y\right] /\left[\mathbb{E}\left[L_{0} \mid X_{0}=0\right] p(0)+\right.$ $\left.\int_{0}^{t_{p}} \mathbb{E}\left[L_{0} \mid X_{0}=y\right] p(y) d y\right]$. The ergodic measure $p()$ of this Markov chain is in fact the stationary age distribution $\tilde{f}()$ as defined by Berg and Epstein (1976).

Since $p(0)+\int_{0}^{t_{p}} p(y) d y=1, \mathbb{E}\left[C_{0} \mid X_{0}=0\right]=c_{p}+c_{f} M_{0}(\Delta), \mathbb{E}\left[C_{0} \mid X_{0}=y\right]=c_{f} M_{y}(\Delta), \mathbb{E}\left[L_{0} \mid X_{0}=y\right]=\Delta$, and $p()=\tilde{f}()$, we have $\lim _{t \rightarrow \infty} \mathbb{E}[C(t)] / t=\left[c_{f}\left[M_{0}(\Delta) p(0)+\int_{0}^{t_{p}} M_{y}(\Delta) p(y) d y\right]+c_{p} p(0)\right] / t$, which is Equation (11). It is worth mentioning that the one-step transition kernels of the Markov chain of ages, given below, are also based on the renewal density function.For $t_{p} \leq \Delta$ :

$$
\begin{aligned}
& p(x, 0)=R(\Delta \mid x)+\int_{t_{p}}^{\Delta} m_{x}(\Delta-u) R(u) d u, \\
& p(x, y)=m_{x}(\Delta-y) R(x) .
\end{aligned}
$$

For $t_{p}>\Delta$ :

$$
\begin{aligned}
p(x, 0) & =0, & 0 \leq x<t_{p}-\Delta, \\
p(x, 0) & =R(\Delta \mid x), & t_{p}-\Delta \leq x<t_{p}, \\
p(x, y) & =m_{x}(\Delta-y) R(x), & 0<y \leq \Delta, \\
p(x, x+\Delta) & =R(\Delta \mid x) . &
\end{aligned}
$$

\section{Generalization of the Calendar-based Age Replacement Policy}

In this section, we first discuss several variations of the calendar-based age replacement policy where the result of Theorem 1 can be generalized. We then show how the Markov renewal technique can be used to analyze the grouped modified block replacement policy where Scarf and Deara (2003) stated there is no closed-form expression for its cost per time unit.

Prolonged Downtime In the calendar-based age replacement policy, the component is immediately replaced with a new one at failure. However, if the failure happens just before one of the fixed checking points, it might be more reasonable to delay the failure renewal until the scheduled checking point. Let us consider a new policy that adjust the calendar-based age replacement policy by postponing the failure replacement to the next checking point if the failure occurs within $\left(n \Delta-t_{d}, n \Delta\right), n \geq 1$. Further let $c_{d}$ denotes the downtime cost per time unit in case the failure renewal is delayed. By defining $Y_{n}$ as the forward time for the new policy where $0 \leq Y_{n}<\Delta-t_{d}$, 
the long-run expected cost per time unit can be derived in terms of the expected cost and length of one renewal cycle and the probability measure of the chain $\left\{Y_{n}\right\}$.

Alternating Fixed Checking Intervals One of our assumptions in the calendar-based age replacement policy is that the length of the checking intervals is constant being equal to $\Delta$. However, in some cases, it may be more realistic to have unequal checking intervals due to weather conditions and/or geographical locations. For example, it might be hard to check the ages of wood poles installed in far locations in winter. Therefore, a periodic schedule of checking the ages at the beginning of, for example, March and July is more practical. In this case, the length of the checking intervals alternates between four and eight months. Let us now formally consider an extension of the calendar-based age policy where the length of checking intervals alternates between short and long values being equal to $\Delta_{s}$ and $\Delta_{l}$, respectively.

Let us further define $\left\{\left(X_{n}, Y_{n}\right): n=0,1,2, \ldots\right\}$ to be a two-dimensional homogeneous Markov chain with state space $S=\left[0, \max \left(\Delta_{s}, \Delta_{l}\right)\right) \times\{0,1\}$. In this policy, $X_{n}$, similar to the calendar-based age replacement policy, is the $n$-th forward time being equal to the time between the previous checking point and the $n$-th renewal. The type of the previous checking interval at the $n$-th renewal is denoted by $Y_{n}$ being equal to 0 if it is short and 1 otherwise. The states $(0,0),(0,1),(x, 0)$, and $(x, 1)$ where $0<x<\Delta$ represent, in respective terms, the preventive renewal at the short interval, the preventive renewal at the long interval, the failure renewal in the long interval, and the failure renewal in the short interval.

Defining the preventive renewal at the short interval as the regeneration point and letting $A=\{(0,0)\}, \varphi((0,0))=1, n_{0}=1$, and $0<\lambda \leq R\left(t_{p}+2 \Delta_{s}\right)$, it can be proved that the chain $\left\{\left(X_{n}, Y_{n}\right)\right\}$ is ergodic. The expected cost per time unit over an infinite horizon is given below where $C_{0}$ and $L_{0}$ are the random cost and length of the first renewal cycle as defined in Section 2 and $\pi(x, y)$ is the ergodic measure.

$$
\lim _{t \rightarrow \infty} \frac{\mathbb{E}[C(t)]}{t}=\frac{\sum_{i=0}^{1} \mathbb{E}\left[C_{0} \mid X_{0}=(0, i)\right] \pi(0, i)+\int_{0}^{\Delta_{l}} \mathbb{E}\left[C_{0} \mid X_{0}=(y, 0)\right] \pi(y, 0) d y+\int_{0}^{\Delta_{s}} \mathbb{E}\left[C_{0} \mid X_{0}=(y, 1)\right] \pi(y, 1) d y}{\sum_{i=0}^{1} \mathbb{E}\left[L_{0} \mid X_{0}=(0, i)\right] \pi(0, i)+\int_{0}^{\Delta_{l}} \mathbb{E}\left[L_{0} \mid X_{0}=(y, 0)\right] \pi(y, 0) d y+\int_{0}^{\Delta_{s}} \mathbb{E}\left[L_{0} \mid X_{0}=(y, 1)\right] \pi(y, 1) d y}
$$

The above result can be easily extended to a case with $k>2$ different alternating checking point intervals.

Random Checking Intervals In the calendar-based age replacement policy and in the policy with alternating fixed checking intervals, the schedule of checking points is fixed in advance and is independent of the failure history of the component. Relaxing the first assumption allows modeling a more general case where, for example, the checking intervals randomly alternates between short and long intervals. Consider a policy where the length of the next checking interval is random. The probability that it is short (long) with length $\Delta_{s}\left(\Delta_{l}\right)$ is $q(1-q)$, independent of the previous intervals. By constructing a two-dimensional Markov chain as discussed above, it can be shown that the expected cost per time unit for an infinite time span is represented in terms of the ergodic measure of the Markov chain and the expected cost and length of one renewal cycle. 
Grouped Modified Block Replacement (Policy IVa2 of Scarf and Deara 2003) A two-component system with type I failure dependence is considered where the failure of component 1 (2) induces a failure of component $2(1)$ with probability $p(q)$ and has no effect on component 2 (1) with probability $1-p(1-q)$. The system is considered failed if at least one of the components has failed. Under this policy, we replace the failed component(s) on the failure of the system. At fixed checking points that are scheduled in $\Delta$ time units, we also preventively replace components 1 and 2 if their respective ages are greater than their critical replacement ages $t_{p}^{1}$ and $t_{p}^{2}$.

To formally analyze this policy, we define $\left\{\left(X_{n}, Y_{n}, A_{n}\right): n=0,1,2, \ldots\right\}$ to be a three-dimensional homogeneous Markov chain where $0 \leq X_{n}<\Delta, Y_{n} \in\{0,1,2\}$ and $0 \leq A_{n} \leq \Delta+\max \left(t_{p}^{1}, t_{p}^{2}\right)$. In this policy, $X_{n}$ is the $n$-th forward time. If both components are replaced at the $n$-th renewal, $Y_{n}=0$, if only component 1 is replaced, $Y_{n}=1$, and if only component 2 is replaced, $Y_{n}=2$. The age of the component that is not replaced at the $n$-th renewal is denoted by $A_{n}$. The states $(0,0,0)$, $(x, 0,0),(x, 1, a)$, and $(x, 2, a)$ where $0<x<\Delta$ and $a>0$ represent the preventive renewal of both components at fixed checking point, the failure renewal of both components, the failure renewal of component 1 where component 2's age is $a$, and the failure renewal of component 2 where the component 1 's age is $a$, respectively.

Following the same analysis as in the calendar-based age replacement policy, it can be proved that the preventive renewal of both components, i.e., the state $(0,0,0)$, is the regeneration point and the cost per time unit ratio can be written using the ergodic measure of $\left(X_{n}, Y_{n}, A_{n}\right)$ chain and expected cost and length of one renewal cycle. We think that the same three-dimensional Markov chain can be used to analyze opportunistic grouped modified block replacement policy (policy IVc2) which Scarf and Deara (2003) analyzed using simulation. The Markov renewal technique can also be applied to analyze other block and age-based replacement policies developed by Scarf and Deara $(1998 ; 2003)$ for a two component system with failure dependency. This technique will likely result in simpler expressions for long-run expected cost per time unit whose calculations are easier and faster. 